\title{
The Beginning of the End of Frustration
}

\author{
T. JACKS \\ Department of Biology, Howard Hughes Medical Institute, Center for Cancer Research, Massachusetts Institute of \\ Technology, Cambridge, Massachusetts 02139
}

\begin{abstract}
The 70th Cold Spring Harbor Symposium, entitled "Molecular Approaches to Controlling Cancer," represented a critical and exciting transition in the fields of cancer research and cancer control. After years of successful research into the molecular genetic and cellular defects that underlie the disease, anticancer agents developed on the basis of these insights are now being used regularly in the treatment of cancer patients. Based on the availability of many more experimental drugs, powerful new methods of analysis, accurate models of the disease, and novel insights into cancer-relevant pathways that affect disease initiation and progression, there is considerable reason for optimism in the ability to one day control cancer.
\end{abstract}

The 70th Cold Spring Harbor Symposium was the fourth in this illustrious series that has focused on cancer. The last time the Symposium was dedicated to this disease and its research sphere was in 1994. My counterpart for that edition was Ed Harlow, who wrote in the introduction of his summary that despite impressive advances in our understanding of the molecular underpinnings of cancer, our ability to treat or otherwise control the disease had not substantially improved. He concluded that it was a "frustrating time" (Harlow 1994).

In the ensuing 11 years, times have changed. The 70th Symposium was held in early June 2005. Entitled "Molecular Approaches to Controlling Cancer," it marked a turning point in this field. After decades of painstaking research aimed at understanding the genes that go awry in the development of cancer and the cellular and physiological processes that are affected in the progression of the disease, the field of molecular oncology is - finally - having an impact on the treatment of patients with cancer. For the more than 500 attendees of the 70th Symposium, the sense of accomplishment was palpable, and there were tangible examples of success. Indeed, in the last half-dozen years, several new anticancer agents, borne from our improved understanding of the molecular biology of cancer, have been approved for use in humans. These agents are changing the lives of cancer patients through prolongation of life, reduced side effects of treatment, and, in some cases, cures.

As reviewed at the Symposium, substantial new progress in the rational development of new drugs for cancer, as well as critical new insights into the biology of the disease, have led to the realistic sense that the control of cancer is within reach. Moreover, with a remarkably powerful new tool kit composed of genomic information and sophisticated methods to interrogate cells and organisms, the pace of discovery has noticeably hastened. The field of cancer science has matured a great deal over the years. One now sees regular examples of the continuum of basic research and clinical investigation, with the latter

All authors cited here without dates refer to papers in this volume. increasingly including molecular measurements and other detailed analyses of diseased tissue. This more integrated approach to cancer research, cancer drug development, and clinical investigation is essential to achieve our common goal.

Not surprisingly, the 66 oral presentations and numerous posters at the 70th Symposium covered considerable ground. The papers contained within this volume provide a detailed description of the science described in six days of oral presentations. I do not try to represent them fully here and, instead, only touch on some important themes that emerged over the course of an exhilarating (and exhausting) week. Unfortunately, I will not be able to adequately capture the feeling of hope that emerged from this collection of talks and posters. Suffice it to say that we are witnessing the beginning of the end of frustration.

\section{CHARACTERIZING CANCER DRUG SENSITIVITY AND RESISTANCE}

The era of targeted therapies for cancer is now well under way with drugs such as Gleevec, Herceptin, Iressa, Tarceva, Avastin, and others now used widely to treat various tumor types. The efficacy of this new generation of anticancer agents provides the basis for much of the enthusiasm for our ability to effectively counteract the effects of molecular alterations in developing tumors. However, although it is well established when and where these agents should be prescribed in some cases, considerable work remains to completely define the specific molecular context that confers drug sensitivity and resistance. To fully realize the power of personalized medicine for cancer, it will be necessary to match the genotype of the tumor (as well as other associated individual characteristics) to the appropriate agent or agents that have the best chance of eradicating it. In many cases, this will involve the use of targeted anticancer drugs in combination with conventional chemotherapeutics or radiation. Beyond establishing the hallmarks of initial sensitivity, it is also essential that we continue to investigate the mechanisms of acquired drug resistance. As with conventional anticancer agents, the use of targeted agents leads eventually to re- 
sistant tumors and relapse. The mechanisms of resistance are being elucidated in a small number of cases, but this remains a critical area of study.

Two sets of examples illustrate the state of the field in this area. The most completely understood exploration of sensitivity/resistance involves Gleevec and the treatment of chronic myelogenous leukemia (CML). Sawyers and colleagues have demonstrated that leukemias that are initially sensitive to treatment by virtue of the presence of a Bcr-Abl fusion oncoprotein can achieve resistance through Abl kinase domain mutations that affect drug binding. Fortunately, a new class of Abl kinase inhibitors has proven to be effective in treating this category of Gleevec-resistant CML (Sawyers). The use of the epidermal growth factor receptor (EGFR) inhibitors Iressa and Tarceva in the treatment of lung cancer has generated considerable interest and debate in the past year, and several speakers discussed their findings in this area. Whereas it is clear that mutations in EGFR in some forms of non-small-cell lung cancer confer sensitivity to these drugs (Varmus et al.; Thomas et al.), additional data described by Hillan (Amler et al.) and Haber (Haber et al.) indicate that other alterations (including EGFR gene amplification) are associated with sensitivity as well. Moreover, reminiscent of the Gleevec experience, resistance to Iressa/Tarceva appears to be commonplace. One form of resistance is associated with a second-site mutation in EGFR, and this may be amenable to second-generation small-molecule inhibitors (Varmus et al.; Haber et al.). However, based on cell culture models, extragenic mutations can also confer resistance to Iressa/Tarceva (Haber et al.).

Several presentations heralded the next phase of targeted anticancer agents. Ashworth and colleagues have developed a strategy to take advantage of the DNA repair deficiency of cancer cells mutant for BRCA1/2 (Tutt et al.). They have demonstrated that such cells are exquisitely sensitive to inhibition of the DNA repair-associated enzyme PARP, thus revealing a potential therapeutic window for the treatment of BRCA1/2 mutant breast and ovarian cancers. In the very promising area of antiangiogenesis therapy, for which clinical validation has already come from the use of Avastin, additional agents and strategies are rapidly emerging. Yancopolous described the further development of the "VEGF Trap" method of VEGF inhibition, including promising preclinical and early clinical data (Rudge et al.). Moreover, he discussed the importance of understanding the specific context that determines initial tumor responsiveness and resistance to therapy. Benezra and colleagues have substantially advanced the study of Id proteins in establishing and maintaining tumor vasculature, now showing that Id gene mutations coupled with the use of the HSP90 inhibitor 17AAG block tumor development in a mouse model of breast cancer (Benezra et al.). As reviewed by Kaelin, McCormick, Workman, and others (Kaelin; Rodriguez-Viciana et al.; Workman), additional kinase inhibitors are showing promise in different phases of clinical testing. These authors all emphasized the importance of matching the inhibitor to the genetics and biology of the treated tumor. Finally, Lane and Adams discussed new agents that target key factors in cell death regulation (p53 and the Bcl-2 family), which will broaden the range of therapeutic opportunities (Lane; Adams).

\section{MOUSE MODELS OF CANCER COME OF AGE}

A large percentage of the presentations at the 70th Symposium highlighted the use of genetically engineered mouse models of cancer. It is quite clear that thoughtful preclinical testing - of hypotheses as well as anticancer agents - is a key step in refining concepts of how cancers arise and progress and how to best treat them. Multiple presentations illustrated the power of such models in addressing critical questions, such as the cells of origin of cancer (Parada et al.; Kim et al.; Lawson et al.; P. Beachy, unpubl.), mechanisms of tumor progression (Calbó et al.; Tward et al.; Simin et al.; Tonon et al.), modes of cancer drug sensitivity and resistance (Varmus et al.; Zender et al.; Barbacid et al.), key cancer genetics pathways (Sherr et al.; Grisendi and Pandolfi; Tuveson and Hingorani; Evan et al.; Iaquinta et al.; M. Serrano, unpubl.), and how to match the biology of the tumor with its treatment (Benezra et al.; Folkman and Ryeom; D. Hanahan, unpubl.). Also noteworthy was the increasing attention paid to comparative analysis of the tumor development and cancer genetics in humans and in cognate mouse models. The use of these models will undoubtedly refine and, in some cases, define methods of detecting cancer, preventing tumor development and spread, and eliminating advanced disease.

\section{CONSIDERING CANCER IN CONTEXT}

Cancer cells develop in the context of normal cells and, throughout their progression to metastasis, have intimate and essential interactions with the cells that surround them. This more tissue-oriented view of cancer is important not only for shaping our thinking about the factors that govern tumor development but also for identifying novel opportunities for intervention. The conceptualization of cancer as an aberrancy of normal development and tissue homeostasis is centuries old; however, the methods to investigate these critical interactions and visualize the many normal cell types that affect incipient cancer cells have been developed rather recently. Numerous speakers focused on the role of cell-cell interactions and non-tumorcell-autonomous factors that can influence the transformation process and/or participate in tumor progression.

Pioneering work from Bissell and colleagues has emphasized the importance of developmental context and the role of cell-cell and cell-matrix adhesion in determining the response to oncogenic stimuli (Bissell et al.). Thus, the setting in which a cell finds itself - whether it be in two- or three-dimensional structures in culture or in the midst of a tissue in vivo - can have a profound effect on how it manifests its genotype. The use of three-dimensional cell culture models has also been exploited by Brugge and coworkers to explore pathways that stimulate cell proliferation and survival (J. Brugge, unpubl.). This system has yielded a number of important findings that address how 
cells overcome their contextual signals in the process of tumor initiation. Infiltrating cells of the immune system, vasculature, and other stromal elements represent additional key players in the biology of cancer, and the molecular and cellular mechanisms that underlie their involvement in this process are being realized at an impressive pace (Egeblad et al.; Grant and Kalluri; Rudge et al.; Folkman and Ryeom; Kaelin; P. Gupta et al.; D. Hanahan, unpubl.). As with tumor angiogenesis, many of these interactions may furnish the basis for powerful new anticancer strategies.

The relationship between tissue homeostasis and cancer development has been enhanced further with recent evidence linking tissue stem cells with the early stages of tumorigenesis. For example, as discussed by Morrison (Pardal et al.) and M. van Lohuizen (unpubl.), pathways that are important in stem cell self-renewal are commonly targeted in cancer. In addition, various studies using mouse models of cancer have implicated stem/progenitor cells as the cell of origin of different tumor types (Lawson et al.; Parada et al.; Kim et al.). These studies do not rule out the possibility that more differentiated cells can undergo transformation and participate in tumor initiation as well. However, the inherent properties of stem cells make them particularly attractive targets. Studying the descendants of these abnormal cells in otherwise normal tissue can lead to the identification of cells with features of preneoplasia, as discussed by Tlsty (Berman et al.). The identification of the cell type(s) that seeds tumors is important for our basic understanding of the origins of the process and will certainly have implications for early detection and chemoprevention of cancer.

The vast majority of cancer deaths are attributable to the effects of metastasis, and yet we still know rather little about this process. Recent progress is encouraging, however. As with tumor initiation, close analogies with normal development and the importance of cell-cell interactions are evident regarding metastasis as well. Weinberg and colleagues have performed a series of experiments recently that focus on the transcriptional control of the epithelial-mesenchymal transition (EMT) during metastatic spread (P. Gupta et al.). The EMT occurs during gastrulation and at other developmental stages, and it has been linked to the propensity of tumor cells to migrate, invade, and remodel the extracellular matrix. Massagué has used transcriptional profiling to identify a series of genes that act in concert to influence the tissue destination of tumor cells and their behavior once there (G. Gupta et al.). Given that the metastatic process may initiate very early in tumor progression, the inhibition of metastasis per se will be challenging. However, with greater insight into the biology of metastatic cells, it may be possible to ameliorate the tissue-damaging effects of established metastases.

\section{CANCER RESEARCH IN THE POST-GENOMIC ERA}

Another notable change in the nature of cancer research as represented at the 70th Symposium was the reliance on whole-genome analysis. The sequencing of the genomes of humans and many experimental organisms has provided a wealth of new information and a remarkable series of new analytic and experimental tools. Powerful insights into the biology of cancer cells and potential new targets of therapy are emerging from in-depth analysis of the sequence, structure, and expression state of large collections of genes in human tumor cells and those from mouse models (Garraway et al.; Futreal et al.; Thomas et al.; Tonon et al.; Hicks et al.; J. Gray, unpubl.). The epigenetic state of the cancer genome is another rich source of information about gene regulation in tumors, and the pathways that govern chromatin modulation in tumorigenesis are potential targets of therapy (Baylin and Chen; Berman et al.; Herman; Feinberg). Likewise, understanding the mechanisms that govern chromosome integrity and how these processes are subverted in cancer is an essential component of a more integrated view of molecular oncology (de Lange; Galloway et al.; Armanios and Greider; J. Campisi, unpubl.).

The work of Futreal and colleagues at the Wellcome Trust Sanger Institute on the sequencing of the entire protein kinase family in a series of human cancer samples and cell lines engendered considerable discussion. Although it is clear that some somatically acquired mutations can be identified through such methods and that these represent attractive targets for anticancer therapy, for most tumors studied, the frequency of mutation was quite low (Futreal et al.). On the other extreme, a few samples exhibited a remarkably high mutation frequency, perhaps suggesting prior exposure to a powerful mutagen or a defect in one or more DNA repair mechanisms (Futreal et al.). In this context, it would be difficult to distinguish the cancer-relevant mutations from the random mutations. During a special session on the proposed joint National Cancer Institute-National Genome Research Institute effort to sequence large gene collections from selected human tumors, there was a spirited discussion of the likely outcomes given the Sanger Institute experience. Moreover, in times of tightening federal budgets for cancer research, many participants questioned the wisdom of very large scale spending on such an initiative, especially when so many known cancer genes and pathways remain incompletely understood. Still, given the potential for short-term benefit in terms of identifying druggable targets for cancer therapy, it seems prudent to undertake a series of pilot projects involving sequencing up to a few thousand genes from a limited number of well-annotated tumor samples; ideally, these same samples would also be subjected to other forms of genomic analysis. The information gained from these pilot studies would then determine whether a larger-scale effort is warranted.

Genome-scale functional analysis and sophisticated, RNAi-based genetic screens described at the Symposium provided a glimpse into an exciting new direction in cancer research. Using collections of cDNAs (Pearlberg et al.; J. Brugge, unpubl.), shRNA libraries (Westbrook et al.; G. Hannon; R. Bernards; both unpubl.), or combinations of siRNAs and chemotherapeutic drugs (Friend), several speakers discussed the power of large-scale, cellbased screens to modulate cancer phenotypes or path- 
ways. Such screens will also be key to discovering genes and pathways on which cancer cells are specifically dependent, with or without exposure to therapeutic agents.

\section{LOOKING AHEAD}

The 70th Cold Spring Harbor Symposium was an inspiring moment in the history of cancer research and cancer care. We are now witnessing the steady approval of molecularly targeted agents for the treatment of the disease. The dramatic improvement in the methods to model cancer and the emerging arsenal of tools and techniques to study tumors with unprecedented precision will yield many more therapeutic agents in the years to come. These same methods - applied to models of the disease and, very importantly, human clinical specimens - will guide the use of the proper agents in individual patients through an understanding of the basis of drug sensitivity and resistance. This process will lead to more durable responses in the clinic and, increasingly, cures for the disease.
Much work remains, and many critical facts await discovery. For example, subjects that were presented in a more limited fashion at the Symposium, such as cancer immunology (J. Allison, unpubl.), cancer cell metabolism (Thompson et al.; Wallace), and the role of microRNAs in cancer (G. Hannon, unpubl.), are likely to be critical for our understanding of cancer progression and treatment. However, unlike 11 years ago when Ed Harlow described the frustrating disparity between advances in cancer research and the state of the art in cancer treatment, the tide is now beginning to turn. It would be irresponsible to put a date on when we will finally conquer cancer in all of its manifestations. However, before the next Cold Spring Harbor Symposium on this topic, many battles will be won, and the end of the war will be well within sight.

\section{REFERENCE}

Harlow E. 1994. An introduction to the puzzle. Cold Spring Harbor Symp. Quant. Biol. 59: 709. 


\section{$8_{8}^{\infty} \mathrm{CSH} \&$ Cold Spring Harbor Symposia SYMPOSIA on Quantitative Biology}

\section{The Beginning of the End of Frustration}

\section{T. JACKS}

Cold Spring Harb Symp Quant Biol 2005 70: 525-528

Access the most recent version at doi:10.1101/sqb.2005.70.053

References This article cites 1 articles, 1 of which can be accessed free at: http://symposium.cshlp.org/content/70/525.full.html\#ref-list-1

\section{License}

Email Alerting Receive free email alerts when new articles cite this article - sign up in Service the box at the top right corner of the article or click here. 